OPEN ACCESS

Edited by:

Meixiang Zhang,

Nanjing Agricultural University,

China

Reviewed by:

Qiong Zhang,

University of California,

Berkeley, United States

Biao Gu,

Northwest $A$ and F University, China

*Correspondence:

Zhao Zhang

zhangzhao@cau.edu.cn

Specialty section:

This article was submitted to Plant Pathogen Interactions,

a section of the journal

Frontiers in Plant Science

Received: 09 July 2021 Accepted: 24 August 2021 Published: 01 October 2021

Citation:

Tian Y, Zhang S, Liu X and Zhang $Z$ (2021) Global Investigation of TBL Gene Family in Rose (Rosa chinensis) Unveils RcTBL16 is a

Susceptibility Gene in Gray Mold

Resistance.

Front. Plant Sci. 12:738880 doi: 10.3389/fpls.2021.738880

\section{Global Investigation of TBL Gene Family in Rose (Rosa chinensis) Unveils RcTBL16 Is a Susceptibility Gene in Gray Mold Resistance}

\author{
Yu Tian, Shiya Zhang, Xintong Liu and Zhao Zhang* \\ Beijing Key Laboratory of Development and Quality Control of Ornamental Crops, Department of Ornamental Horticulture, \\ China Agricultural University, Beijing, China
}

The TRICHOME BIREFRINGENCE-LIKE (TBL) family is an important gene family engaged in the $\mathrm{O}$-acetylation of cell wall polysaccharides. There have been a few reports showing that TBL participated in the resistance against phytopathogens in Arabidopsis and rice. However, no relevant studies in rose (Rosa sp.) have been published. In this study, a genome-wide analysis of the TBL gene family in rose was presented, including their phylogenetic relationships, gene structure, chromosomal positioning, and collinearity analysis. The phylogenetic analysis revealed a total of 50 RcTBL genes in the rose genome, and they are unevenly distributed across all seven chromosomes. The occurrence of gene duplication events suggests that both the whole genome duplication and partial duplication may play a role in gene duplication of RcTBLs. The analysis of $\mathrm{Ka} / \mathrm{Ks}$ showed that the replicated RCTBL genes underwent mainly purifying selection with limited functional differentiation. Gene expression analysis indicated that 12 RcTBLs were down-regulated upon the infection of Botrytis cinerea, the causal agent of the gray mold disease of rose. These RcTBLs may be a sort of candidate genes for regulating the response of rose to $B$. cinerea. Through virus-induced gene silencing, RcTBL16 was shown to be associated with susceptibility to gray mold in rose. Through this study, meaningful information for further studies on the function of the TBL protein family in rose is provided.

Keywords: Rosa sp., TBL, Botrytis cinerea, gene family, expression, S-gene

\section{INTRODUCTION}

The cell wall is particularly important in plant growth and development because it maintains the form of the plant cell, allows intercellular communication, responds to external environmental variables, and interacts with pathogenic microorganisms (Keegstra, 2010; Xin et al., 2010). The plant cell wall has a complicated and dynamic structure, which is mainly composed of polysaccharide polymer, protein and lignin. $O$-acetylation occurs extensively in the plant cell wall, most notably with hemicelluloses, pectins, and lignins. The replacements of $O$-acetyl group usually happen on various specific glycosyl residues of cell wall polysaccharides. In addition, cell wall polysaccharides can be either mono- or diacetylated, and the extent of $O$-acetylation depends on species, tissue type, and growth status of plants. 
The biosynthetic pathway and function of $O$-acetylation of cell wall polysaccharides have not yet been fully understood. Modifications in $\mathrm{O}$-acetylation levels are known to alter plant growth and development, as well as their defense against pathogens, and that this effect is most likely achieved by altering the cell wall structure. The REDUCED WALL ACETYLATION (RWA) protein, as well as the ALTERED XYLOGLUCAN9 protein and TRICHOME BIREFRINGENCE-LIKE (TBL) protein families, has been identified as being involved in the $O$-acetylation pathway of plant cell walls. RWA proteins could be acetyl donor transporters, transporting acetyl CoA into the Golgi apparatus (Manabe et al., 2011, 2013). AXY9 protein may be required for $O$-acetylation of cell wall polysaccharides by producing acetylation intermediates (Schultink et al., 2015).

Unlike the RWA and AXY9 proteins, a number of TBLs have been identified as polysaccharide acetyltransferases, catalyzing the $O$-acetylation of specific cell wall polymers including xylan (Urbanowicz et al., 2015; Zhong et al., 2017a; Zhong et al., 2018a,d), xyloglucan (Zhong et al., 2018c,e; Zhong et al., 2020), mannan (Zhong et al., 2018b), and pectin (Vogel et al., 2004b; Bischoff et al., 2010a; Stranne et al., 2018a; Chiniquy et al., 2019a). TBL proteins have conserved Asp-X$\mathrm{x}$-His (DxxH) motif and Gly-Asp-Ser (GDS) motif that is required for acetyltransferase activity (Bischoff et al., 2010b), since a mutation in either the GDS or DXXH motif could cause TBL proteins to lose their function completely (Zhong et al., 2017a; Zhong et al., 2018e). Studies of tbl mutants in Arabidopsis have demonstrated that dwarfism, stem weakness, and stunted growth of plants are associated with the lack of the TBL genes (Bischoff et al., 2010a; Xiong et al., 2013; Schultink et al., 2015), implying that TBL is critical for plant development. Besides, TBL proteins are related to abiotic stress in plants. Compared with wild-type Arabidopsis plant, the cold tolerance of esk 1 increased significantly (Xin et al., 2010), while tbl10 showed enhanced drought resistance (Stranne et al., 2018a). Furthermore, TBL proteins have also been associated with plant defense against pathogens. The reduction of $O$-acetyl degree of pmr5 in Arabidopsis may lead to its enhanced resistance to powdery mildew (Vogel et al., 2004b; Chiniquy et al., 2019a). According to a recent research, simultaneous mutation of the OsTBL1 and OsTBL2 genes in rice leads to lower acetylation levels and higher vulnerability to leaf blight disease (Gao et al., 2017).

Rose (Rosa sp.) is the most important cut flower in the world, with 8,500 hectares of cut-flower rose cultivation worldwide, with an annual production of over 15 billion stems (Bendahmane et al., 2013), and sales of more than $\$ 11$ billion (Zlesak, 2007). Gray mold caused by Botrytis cinerea is the most devastating disease mainly infecting the flower of rose and affects the production of cut rose all over the world (Gleason and Helland, 2003). The cell wall is the initial barrier that pathogens meet when penetrating the plant, and alterations in cell wall structure might affect the plant's defense against

Abbreviations: hpi, hours post inoculation; ML, maximum likelihood; RPKM, number of reads per kb per million reads; HMM, hidden Markov model; CWI, cell wall integrity; aa, amino acids; VIGS, virus-induced gene silencing. these microbes. O-acetylation is one of the most important modifications of cell wall polymer. TBL proteins, as the main gene family involved in cell wall $O$-acetylation, may influence the resistance or susceptibility of plants to pathogen by varying the degree of cell wall acetylation. However, no research on the function of the TBL gene at the plant genome-wide level has been conducted thus far. We performed the first genomewide analysis of the $R c T B L$ family in rose in this study. Furthermore, a virus-induced gene silencing (VIGS) has confirmed that RcTBL16 was involved in $B$. cinerea susceptibility.

\section{MATERIALS AND METHODS}

\section{Characterization of Putative TBL Proteins in Rose}

We downloaded the complete rose genome sequence and CDS sequence from the website https://lipm-browsers.toulouse.inra. fr/pub/RchiOBHm-V2/ to construct a local genome database. In order to identify non-redundant $R c T B L$ genes in rose genome, the HMM profile of the PC-Esterase domain was obtained

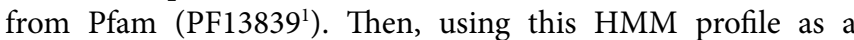
query, by searching the rose genome, all sequences were confirmed to contain a PC-Esterase domain with an E value of $<1 \mathrm{e}^{-3}$ in rose. The distribution of all $R c T B L$ genes on chromosomes was mapped by mapchart 2.2 software.

\section{Gene Structure and Phylogenetic Analyses}

The gene structure map of RcTBL was completed using TBtools (Chen et al., 2018) by means of the rose genome annotation file and protein sequences. Multiple comparisons of RcTBL amino acids (aa) were performed using the ClustalW default parameters. A phylogenetic analysis of RcTBLs then was carried out in MEGA-6.0 software by the maximum likelihood (ML) method, with 1,000 bootstrap replications, JTT with Freqs $(+\mathrm{F})$ model and $50 \%$ partial deletion. Other parameters were set by default. MEGA 6.0 was also applied to construct the unrooted ML trees of TBL proteins from Arabidopsis and rose, the parameter settings being consistent with the separate phylogenetic analysis of RcTBLs.

\section{Collinearity Analyses}

For the purpose of identifying the collinearity of RcTBLs, the genome sequence of rose was downloaded on a local server, and then we used a Multiple Collinearity Scan toolkit (Wang et al., 2012) to determine the microsyntenic relationships between RcTBL genes. Furthermore, collinearity scanning (e-value of $<1 \mathrm{e}^{-10}$ ) was used to evaluate the microsynteny relationships.

\section{Calculation of Ratios of Nonsynonymous (Ka) to Synonymous (Ks) Nucleotide Substitutions}

We used TBtools (Chen et al., 2018) to calculate Ks and Ka nucleotide substitution rates. The $\mathrm{Ka} / \mathrm{Ks}$ ratio of duplicated

${ }^{1}$ http://pfam.xfam.org 
gene pairs was calculated to determine the selection pattern driving the evolution of $R c T B L$.

\section{Expression of RcTBLs in Response to B. cinerea}

RNA-Seq data from rose petals under $B$. cinerea infection were obtained from the National Center for Biotechnology Information database as accession number PRJNA414570 (Liu et al., 2018). The materials for RNA-seq are rose petal discs infected with $B$. cinerea at $30 \mathrm{~h}$ post inoculation (hpi) and $48 \mathrm{hpi}$, with three biological repeats for both infected and control treatments at each time point. Clean sequencing reads were mapped to the Rosa chinensis 'Old Blush' reference genome. ${ }^{2}$ We calculated the gene expression levels of RcTBLs by Reads per kb per million reads and performed a Log2 fold change-based differentially expressed gene analysis by DEseq2.

For validating the RNA-Seq outcomes, RT-qPCR was used to analyze the expression of five $R c T B L$ genes. Total RNA was extracted from rose petals at $30 \mathrm{hpi}$ and $48 \mathrm{hpi}$, respectively. As described previously (Wu et al., 2016), the hot borate method was used to extract total RNA. Then, first-strand cDNA was synthesized using HiScript II Q Select RT SuperMix (Vazyme) in a 20 - $\mu$ l reaction volume with $1 \mu \mathrm{g}$ of DNasetreated RNA. RT-qPCR reaction was run using SYBR Green Master Mix (Takara) and detection was achieved in a StepOnePlus real-time PCR system (Thermo Fisher Scientific). We used RcUBI2 as an internal control and conducted expression analysis using the delta-delta-Ct method of calculation. All primers used as RT-qPCR are listed in Supplementary Table S1.

\section{VIGS and B. cinerea Inoculation Assays}

In order to obtain the pTRV2-RcTBL constructs, a $200 \mathrm{bp}$ fragment from the coding region of RcTBLs was amplified with specific primer pairs and subsequently cloned into the pTRV2 vector (Liu et al., 2002). VIGS was established as previously described (Cao et al., 2019). Briefly, separated petals were obtained from the outermost whorls of cut roses in the second stage of flowering. A $15 \mathrm{~mm}$ disc was then punched from the center of each petal. Agrobacterium tumefaciens consisting of $p T R V 1$ and $p T R V 2$ constructs were mixed in a 1:1 ratio and vacuumed infiltrated into petal discs. Petals were inoculated with $B$. cinerea on day 6 after TRV infection and photographed $60 \mathrm{~h}$ later to obtain images with disease lesions, which were statistically analyzed by ImageJ. Each gene was silenced at least three times with 48 petals as a replicate. Student's $t$ test was performed to determine the significance of lesion size. After photographing, the petal samples were collected for further validating of silencing efficiency by RT-qPCR. The primers used to detect silencing efficiency of RcTBL16 or $R c T B L 35$ are the same primers as those used to detect expression in response to B. cinerea, and listed in Supplementary Table S1.

${ }^{2}$ https://lipm-browsers.toulouse.inra.fr/pub/RchiOBHm-V2/

\section{RESULTS}

\section{Identification of RcTBL Genes in Rose}

As previously stated, the TBL protein family is distinguished by a conserved GDS signature and DXXH motif, as well as an N-terminal transmembrane domain in most of the cases (Bischoff et al., 2010a,b). A total of 61 candidate RcTBL proteins were obtained in rose by the Hidden Markov model (HMM) profile of PC-Esterase domain (PF13839) contained two conservative motifs of the TBL protein family. All candidate sequences less than 150 amino acids and without the complete conserved motifs were removed; finally, we obtained a total of 50 RcTBLs.

All RcTBLs can be mapped to rose chromosomes; we designated the genes RcTBL01 to RcTBL50 according to their chromosome order. The protein length of RcTBLs varies greatly. Of the 50 RcTBLs, RcTBL23 is the longest protein with 630 aa, while the shortest is RcTBL15 with 154 aa. The average length of proteins in RcTBL family is 409 aa. Details of the RcTBL genes, with gene number, chromosomal location, introns, exons, CDS and aa length are listed in Table 1.

\section{Chromosomal Locations, Whole-Genome Duplication, and Microsynteny}

All 50 RcTBL genes were distributed unevenly across seven rose chromosomes (Figure 1), with chromosome 2 having the highest density, gathering 13 RcTBL genes, followed by chromosome 5 with 12 RcTBL genes clustered on it, and chromosome 7 having the fewest $R c T B L$ genes with only three members. We further investigated the duplication events in $R c T B L s$. Tandemly duplicated genes were defined as arrays of two or more homologous genes in the $100 \mathrm{~kb}$ range. Three $R c T B L$ gene pairs were discovered in the rose genome, each on a different chromosome, suggesting that segmental duplication occurs within these regions in rose. Collinearity analyses of the RcTBL genes on the chromosome are depicted in Figure 2.

To explore the selective constraints among duplicated $R c T B L$ genes, the ratio of nonsynonymous (Ka) to synonymous (Ks) nucleotide substitutions (Ka/Ks ratio) of three pairs of duplicated genes (Table 2) was calculated. Typically, $\mathrm{Ka} / \mathrm{Ks}>1$ is consistent with positive selection, while $\mathrm{Ka} / \mathrm{Ks}<1$ indicates purifying selection. $\mathrm{Ka} / \mathrm{Ks}<1$ for all three duplicated gene pairs (Table 2) suggested that the primary driver of gene evolution in the RcTBL family was purifying selection.

\section{Phylogenetic and Gene Structural Analysis of Rose TBL Genes}

A total of 46 TBLs were identified on Arabidopsis thaliana and many of them have been established as $O$-acetyltransferases or potential $O$-acetyltransferase genes (Table 3 ). To evaluate the relationship between the TBL proteins of rose and $A$. thaliana, a compound phylogenetic tree was constructed using the full-length protein sequences of 46 Arabidopsis and 50 rose TBLs by the ML method. We found that most of the Arabidopsis TBL proteins had at least one rose homologue. 
TABLE 1 | Members of the RCTBL gene family as predicted in Rosa chinensis genome sequence.

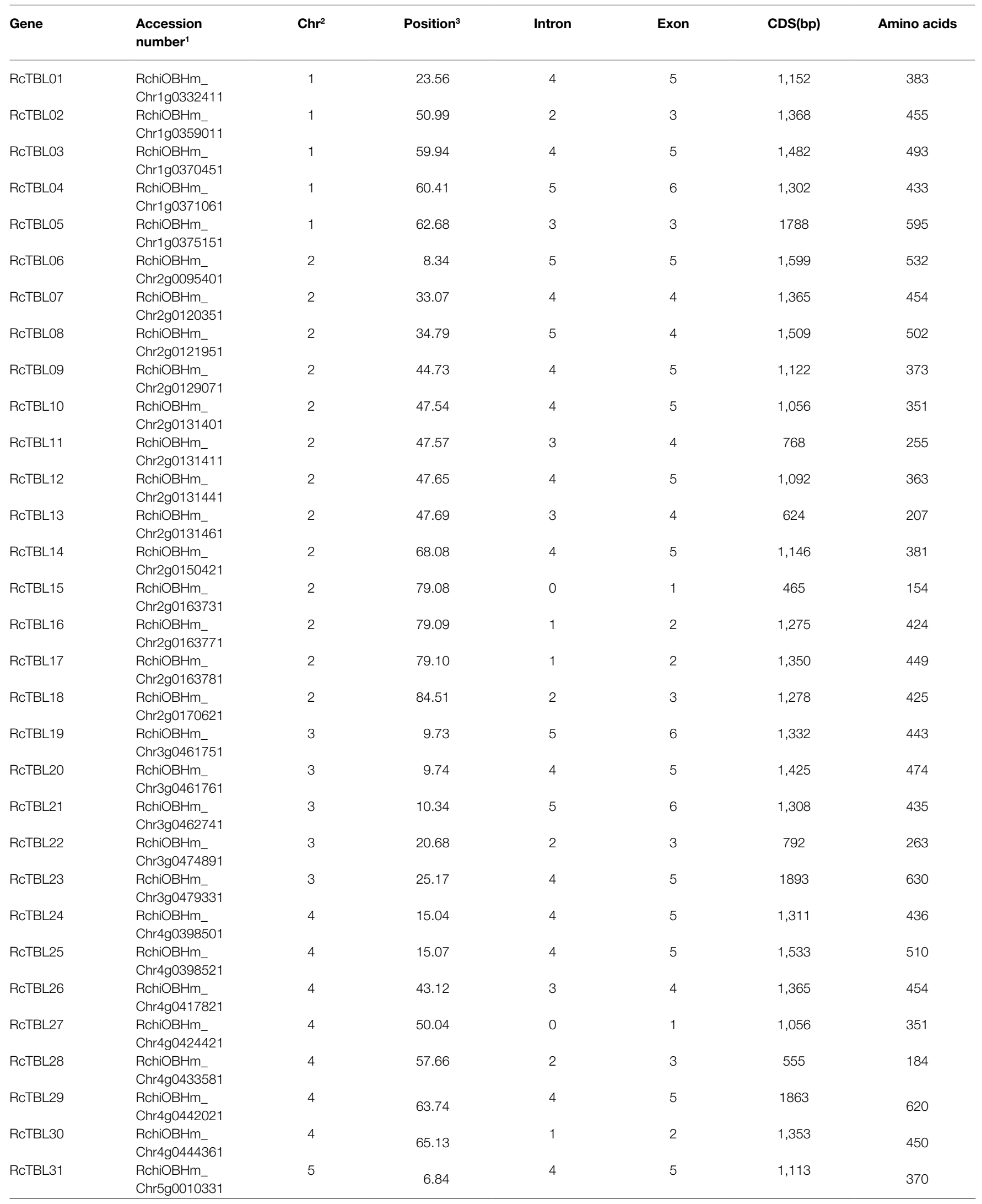


TABLE 1 | Continued

\begin{tabular}{|c|c|c|c|c|c|c|c|}
\hline Gene & $\begin{array}{l}\text { Accession } \\
\text { number }^{1}\end{array}$ & $\mathrm{Chr}^{2}$ & Position $^{3}$ & Intron & Exon & CDS(bp) & Amino acids \\
\hline RcTBL32 & $\begin{array}{l}\text { RchiOBHm_ } \\
\text { Chr5g0025161 }\end{array}$ & 5 & 19.14 & 4 & 3 & 549 & 182 \\
\hline RcTBL33 & $\begin{array}{l}\text { RchiOBHm_ } \\
\text { Chr5g0029581 }\end{array}$ & 5 & 23.19 & 4 & 5 & 1,137 & 378 \\
\hline RcTBL34 & $\begin{array}{l}\text { RchiOBHm_ } \\
\text { Chr5g0029591 }\end{array}$ & 5 & 23.20 & 4 & 5 & 1,023 & 340 \\
\hline RcTBL35 & $\begin{array}{l}\text { RchiOBHm_ } \\
\text { Chr5g0029601 }\end{array}$ & 5 & 23.20 & 4 & 5 & 1,143 & 380 \\
\hline RcTBL36 & $\begin{array}{l}\text { RchiOBHm_ } \\
\text { Chr5g0029611 }\end{array}$ & 5 & 23.21 & 4 & 5 & 1,086 & 361 \\
\hline RcTBL37 & $\begin{array}{l}\text { RchiOBHm_ } \\
\text { Chr5g0031471 }\end{array}$ & 5 & 25.06 & 4 & 5 & 1,251 & 416 \\
\hline RcTBL38 & $\begin{array}{l}\text { RchiOBHm_ } \\
\text { Chr5g0070191 }\end{array}$ & 5 & 76.02 & 4 & 5 & 1,161 & 386 \\
\hline RcTBL39 & $\begin{array}{l}\text { RchiOBHm_ } \\
\text { Chr5g0081011 }\end{array}$ & 5 & 87.06 & 2 & 3 & 549 & 182 \\
\hline RcTBL40 & $\begin{array}{l}\text { RchiOBHm_ } \\
\text { Chr5g0081071 }\end{array}$ & 5 & 87.07 & 5 & 6 & 1,638 & 545 \\
\hline RcTBL41 & $\begin{array}{l}\text { RchiOBHm_ } \\
\text { Chr5g0081121 }\end{array}$ & 5 & 87.16 & 4 & 5 & 1,578 & 525 \\
\hline RcTBL42 & $\begin{array}{l}\text { RchiOBHm_ } \\
\text { Chr5g0081131 }\end{array}$ & 5 & 87.16 & 4 & 5 & 1761 & 586 \\
\hline RcTBL43 & $\begin{array}{l}\text { RchiOBHm_ } \\
\text { Chr6g0247241 }\end{array}$ & 6 & 3.06 & 5 & 6 & 1,260 & 419 \\
\hline RcTBL44 & $\begin{array}{l}\text { RchiOBHm_ } \\
\text { Chr6g0247271 }\end{array}$ & 6 & 3.12 & 6 & 7 & 1,317 & 438 \\
\hline RcTBL45 & $\begin{array}{l}\text { RchiOBHm_ } \\
\text { Chr6g0269281 }\end{array}$ & 6 & 26.54 & 3 & 4 & 1,194 & 397 \\
\hline RcTBL46 & $\begin{array}{l}\text { RchiOBHm_ } \\
\text { Chr6g0277131 }\end{array}$ & 6 & 39.52 & 3 & 3 & 1,323 & 440 \\
\hline RcTBL47 & $\begin{array}{l}\text { RchiOBHm_ } \\
\text { Chr6g0291221 }\end{array}$ & 6 & 54.42 & 3 & 4 & 1,302 & 433 \\
\hline RcTBL48 & $\begin{array}{l}\text { RchiOBHm_ } \\
\text { Chr7g0195391 }\end{array}$ & 7 & 13.24 & 2 & 3 & 1,332 & 443 \\
\hline RcTBL49 & $\begin{array}{l}\text { RchiOBHm_ } \\
\text { Chr7g0217351 }\end{array}$ & 7 & 35.23 & 3 & 4 & 756 & 251 \\
\hline RcTBL50 & $\begin{array}{l}\text { RchiOBHm_ } \\
\text { Chr7g0241111 }\end{array}$ & 7 & 67.11 & 4 & 5 & 1,542 & 513 \\
\hline
\end{tabular}

${ }^{1}$ Available at: https://lipm-browsers.toulouse.inra.fr/pub/RchiOBHm-V2/

${ }^{2}$ Chromosome.

${ }^{3}$ Starting position (Mb).

AtTBL members identified as affecting the $O$-acetylation of xyloglucan, xylan, mannan, and pectin, respectively, were clustered in different branches, suggesting the correctness of our evolutionary tree (Figure 3).

Analysis of the exon-intron structure revealed that the intron structure of RcTBLs were highly variable, ranging from 1 to 6 , with the largest number (23) of RcTBLs containing four introns. In addition, the length of RcTBL introns was extremely varied, ranging from tens to thousands of nucleotides. RcTBL35 contained the longest intron $(4,191 \mathrm{bp})$, while the shortest intron (69bp) was present on RcTBL36 (Figure 4).

The protein sequences of RcTBLs were examined with Pfam and all 50 candidate genes had the PC-esterase domain. Surprisingly, $88 \%$ of RcTBLs possessed a cys-rich domain called the PMR5N domain in Pfam (PF14416) that preceded the PC-esterase domain, implying that it could be a crucial structural element of the TBL family. Furthermore, $64 \%$ of all 50 RcTBL candidates had at least one transmembrane domain, with RcTBL40 having two transmembrane domains, and 18 RCTBLs had no transmembrane domain (Figure 4).

\section{The Expression of RcTBL Genes in Response to $B$. cinerea Infection}

Growing evidence has shown that TBL plays an important role in pathogen defense. In order to investigate the role of RcTBLs in B. cinerea resistance, we obtained RNAseq transcriptomics data from rose petals at $30 \mathrm{hpi}$ (hours postinoculation) and $48 \mathrm{hpi}$ (Liu et al., 2018). The $30 \mathrm{hpi}$ represents the early response to the infection of $B$. cinerea, while the $48 \mathrm{hpi}$ corresponds to the late response. A total of $13 \mathrm{RcTBL}$ genes showed significant changes in expression and, interestingly, they were mainly down-regulated in expression (Table 4). Among them, RcTBL12 and RcTBL35 were both considerably down-regulated at $30 \mathrm{hpi}$ and $48 \mathrm{hpi}$, whereas RcTBL02, RcTBL04, 


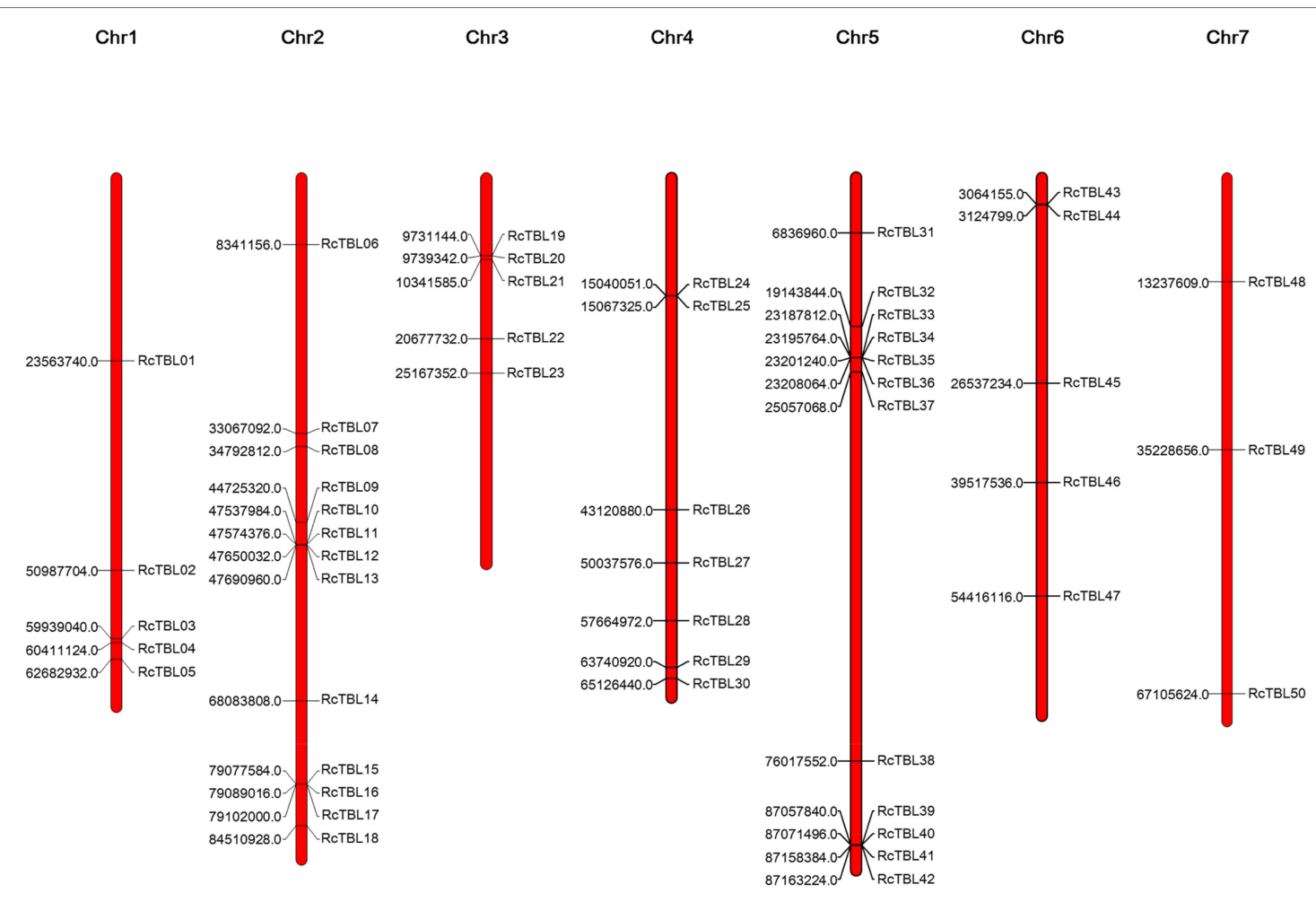

FIGURE 1 | Chromosome localization of rose TBL family members. The distribution of RcTBLs is shown on the seven chromosomes of Rose chinensis. The starting position (bp) of each RcTBL is listed on the left side of the chromosomes.

RcTBL05, RcTBL16, and RcTBL36 were only significantly downregulated at $30 \mathrm{hpi}, R c T B L 23, R c T B L 38$, and $R c T B L 48$ were only significantly down-regulated at $48 \mathrm{hpi}$. Surprisingly, RcTBL18 expression was dramatically decreased at $30 \mathrm{hpi}$ but significantly increased at $48 \mathrm{hpi}$, whereas RcTBL06 and RcTBL09 expression was greatly increased at $48 \mathrm{hpi}$. These genes, which are strongly activated by gray mold, could be crucial in rose resistance to B. cinerea infection. To further validate the RNA-seq expression profile, the expressions of five RcTBLs were examined using RT-qPCR. The RT-qPCR analysis results were found associated with the transcriptome analysis expression patterns (Figure 5).

\section{RcTBL16 Involving in the Defense of Rose Against $B$. cinerea}

The elucidation of gene expression patterns can provide important clues about gene function. Based on the expression results in response to $B$. cinerea, 13 RcTBLs with significant differential expression were considered as potential candidates participating in rose against $B$. cinerea. Their potential role in resistance to this fungus was further illustrated by the reduced expression in rose petals by VIGS. We selected RcTBL16 and RcTBL35 as candidate genes to explore the relationship between TBL family and rose resistance to $B$. cinerea, because (1) apart from RcTBL38 (a PMR5 homolog), RcTBL16 and RcTBL35 were the two of maximum downregulated expressed $R c T B L$ genes in response to $B$. cinerea
(Table 4), and the results of RT-qPCR support this result (Figure 5); (2) both RcTBL16 and RcTBL35 are down-regulated in expression at $30 \mathrm{hpi}$, which represent an early stages of B. cinerea infection (Liu et al., 2018).

In order to silence RcTBL16 and RcTBL35, we cloned approximately $200 \mathrm{bp}$ fragments of them into the $p T R V 2$ vector to generate $p T R V 2-R c T B L 16$ and $p T R V 2-R c T B L 35$. Next, Agrobacterium tumefaciens carrying $p T R V 2-R c T B L 16$, $p T R V 2-R c T B L 35$ and $p T R V 1$ were co-infiltrated into the rose petals to generate silent rose petals. Then, the infiltrated rose petal discs were put on agar medium and inoculated with $B$. cinerea. Comparing the control inoculated with $T R V$-GFP, petals inoculated with $T R V-R c T B L 16$ showed notably reduced disease symptoms, whereas the area of disease spots on petals inoculated with $T R V-R c T B L 35$ had no significantly changes (Figure 6). This result indicated that RcTBL16 is a susceptibility gene involved in Botrytisrose interaction.

\section{DISCUSSION}

$O$-acetylation is a common modification on plant cell walls and is essential for the stability of the polysaccharide network, with a small amount of acetylation modification affecting plant growth and susceptibility to pathogens. TBL is the largest protein family involved in $\mathrm{O}$-acetylation in plants. 


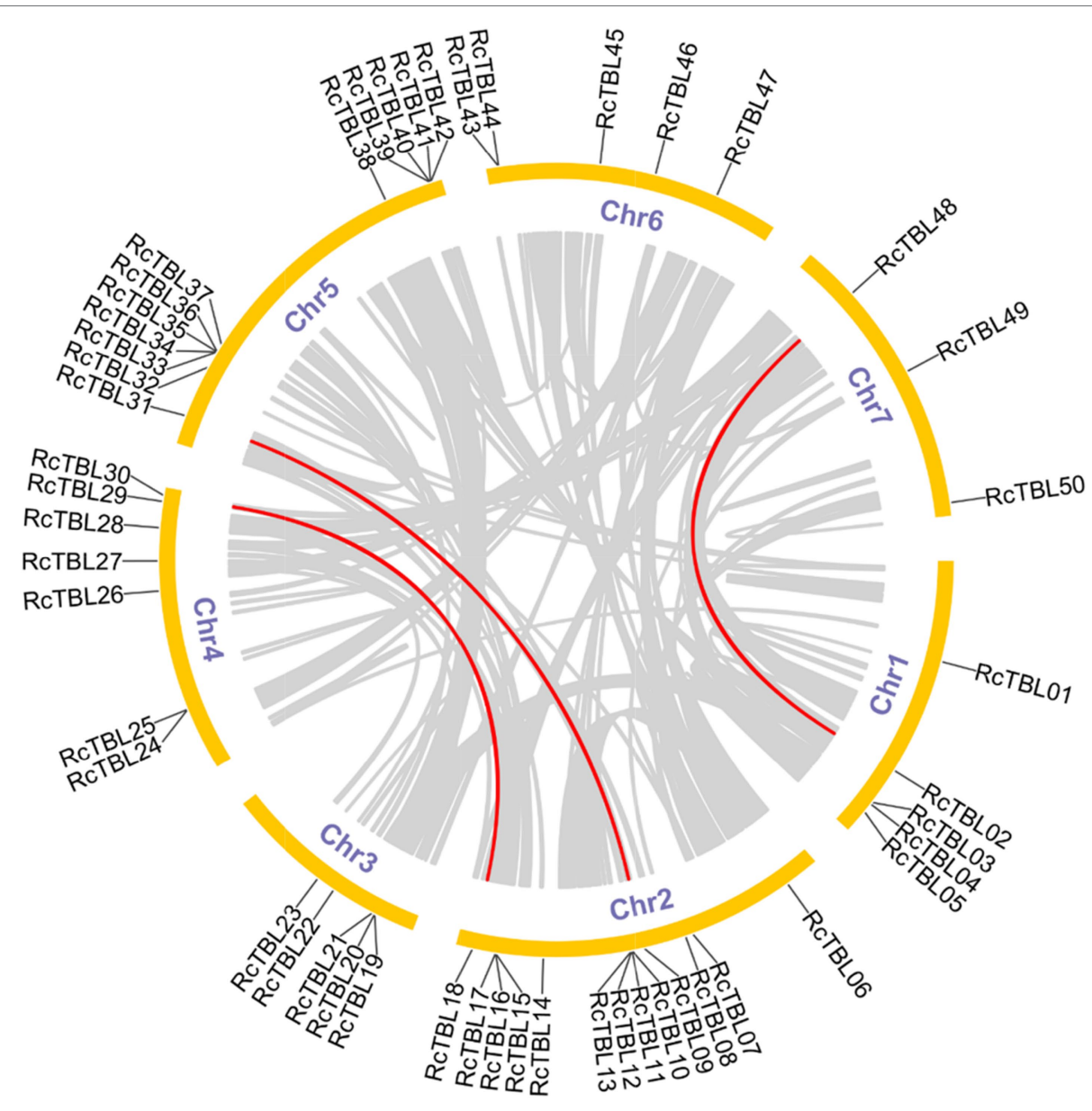

FIGURE 2 | Microsyntenic analyses of the rose TBL protein family members in the $R$. chinensis genome. Circular visualization of rose TBL protein family members is mapped onto different chromosomes by using Circos. The red lines indicate rose TBL genes with a syntenic relationship. The gray lines represent all syntenic blocks in the genome of $R$. chinensis.

TABLE 2 | Duplication analysis of the RCTBL gene family.

\begin{tabular}{|c|c|c|c|c|c|c|c|}
\hline Gene 1 & Gene 2 & $\mathbf{K a}$ & Ks & Ka_Ks & Effective Len & Average S-sites & Average $\mathrm{N}$-sites \\
\hline RcTBL18 & RcTBL30 & 0.645328 & $\mathrm{NaN}$ & $\mathrm{NaN}$ & 1,257 & 275.3333333 & 981.6666667 \\
\hline RcTBL09 & RcTBL31 & 0.327007 & 2.080681 & 0.157164 & 1,107 & 252.4166667 & 854.5833333 \\
\hline
\end{tabular}

There are 46 TBLs in Arabidopsis (Bischoff et al., 2010a), 18 of which have been identified as $O$-acetyltransferases, involved in the $O$-acetylation of xylan, xyloglucan, mannan and pectin respectively (Table 3 ). Several TBL proteins from rice and poplar have also been shown to be xylan $O$-acetyltransferases (Zhong et al., 2018a,d). However, there is still a gap in the comprehensive analysis of the rose TBL gene family, and their function remains to be discovered. Rose genome sequencing project was completed in 2018, enabling genome-wide analysis of the RcTBL gene family. In this study, we have comprehensively analyzed the $R c T B L$ family, including phylogeny, gene structure, chromosomal location, gene duplication events, sequence characterization, and analysis of expression profiles.
We identified 50 rose TBLs, more than Arabidopsis (46) (Bischoff et al., 2010a) but less than poplar (64) (Zhong et al., 2018d), rice (66) (Gao et al., 2017), tomatoes (69) (Zhong et al., 2020), which indicates that TBL protein family has expanded to varying degrees in different plants during the evolution. In our structural analysis, we found that in addition to the PC-esterase domain, RcTBLs have a cys-rich domain, namely PMR5N domain, which may be another vital characteristic of the TBL family. Gene duplication plays a very important role in the expansion of gene families. Checking the phylogenetic relationships of TBL between rose and Arabidopsis showed that most evolved branches contained different amounts of AtTBL and RcTBL proteins, suggesting that the two species displayed conserved evolution. 


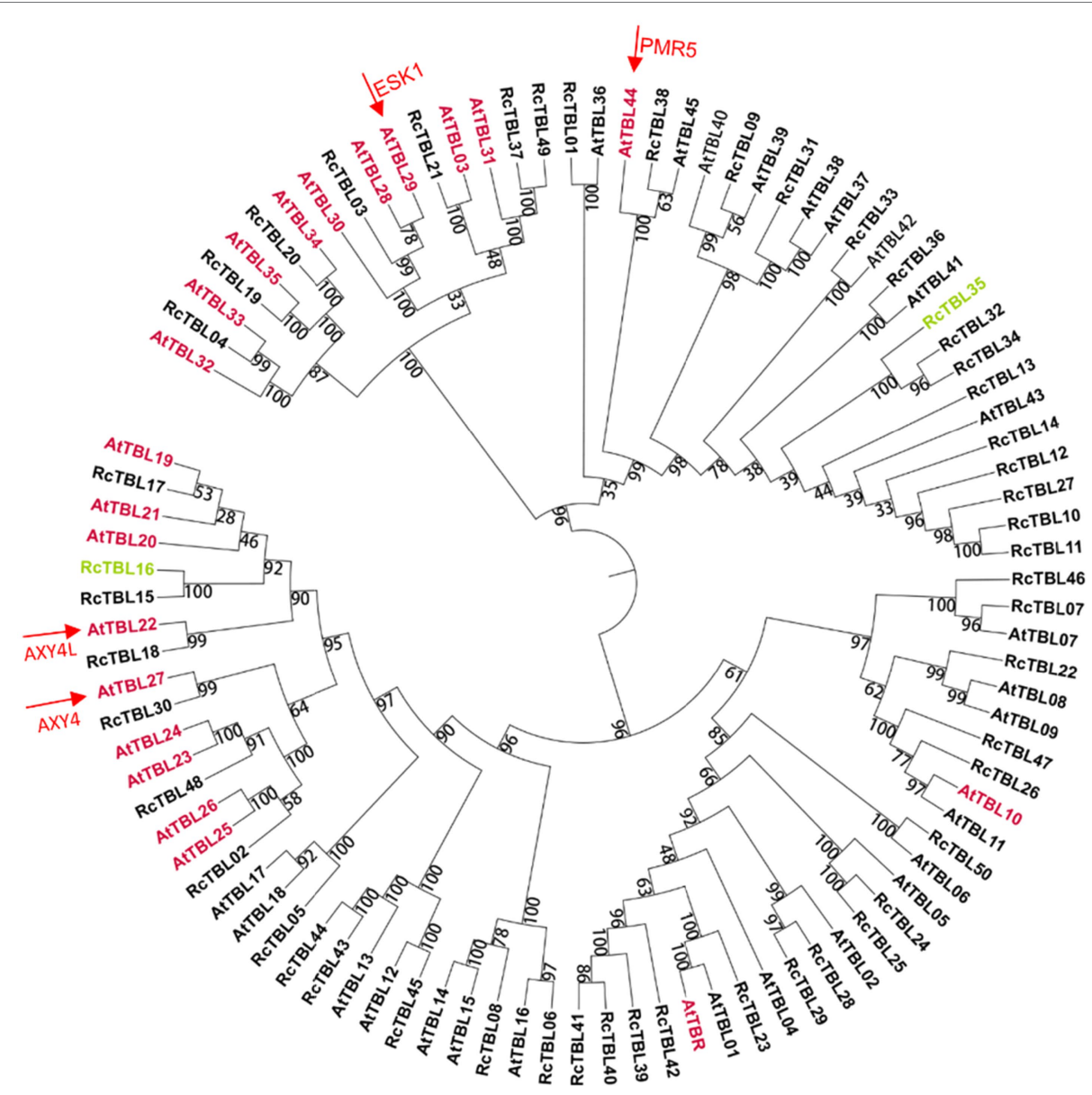

FIGURE 3 | Phylogenetic analyses of TBL protein family in rose with Arabidopsis thaliana TBL protein family. Genes marked with pink have been shown to be involved in $O$-acetylation of cell wall polysaccharides in $A$. thaliana (Table 3); genes marked with green may be involved in the defense of roses against $B$. cinerea and were knocked down by virus-induced gene silencing (VIGS) in rose petals. Arrows refer to the well-known TBL proteins in Arabidopsis. The bootstrap values are indicated on the nodes of the branches.

TABLE 3 | Arabidopsis TBL family genes involved in acetylation.

\begin{tabular}{llll}
\hline Gene name & Gene ID & Polysaccharides & References \\
\hline AtTBL3 & At5G01360 & Xylan & Yuan et al., 2016c \\
AtTBL28 & At2G40150 & Xylan & Zhong et al., 2017a \\
AtTBL29/ESK1 & At3G55990 & Xylan & Xiong et al., 2013 \\
AtTBL30 & At2G40160 & Xylan & Zhong et al., 2017a \\
AtTBL31 & At1G73140 & Xylan & Yuan et al., 2016c \\
AtTBL32 & At3G11030 & Xylan & Yuan et al., 2016a \\
AtTBL33 & At2G40320 & Xylan & Yuan et al., 2016a \\
AtTBL34 & At2G38320 & Xylan & Yuan et al., 2016b \\
AtTBL35 & At5G01620 & Xylan & Yuan et al., 2016b \\
AtTBL19 & AT5G15900 & Xyloglucan & Zhong et al., 2020 \\
AtTBL20 & AT3G02440 & Xyloglucan & Zhong et al., 2020 \\
AtTBL21 & AT5G15890 & Xyloglucan & Zhong et al., 2020 \\
AtTBL22/AXY4L & At3G28150 & Xyloglucan & Gille et al., 2011a \\
AtTBL27/AXY4 & At1G70230 & Xyloglucan & Gille et al., 2011a \\
AtTBL23 & At4G11090 & Mannan & Zhong et al., 2018b \\
AtTBL24 & At4G23790 & Mannan & Zhong et al., 2018b \\
AtTBL25 & At1G01430 & Mannan & Zhong et al., 2018b \\
AtTBL26 & At4G01080 & Mannan & Zhong et al., 2018b \\
AtTBL10 & At3G06080 & Rhamnogalacturonan-I & Stranne et al., 2018b \\
AtTBL44/PMR5 & At5G58600 & Homogalacturonan & Chiniquy et al., 2019b \\
TBR/TBL46 & TBR/TBL46 & Pectin & Bischoff et al., 2010a
\end{tabular}



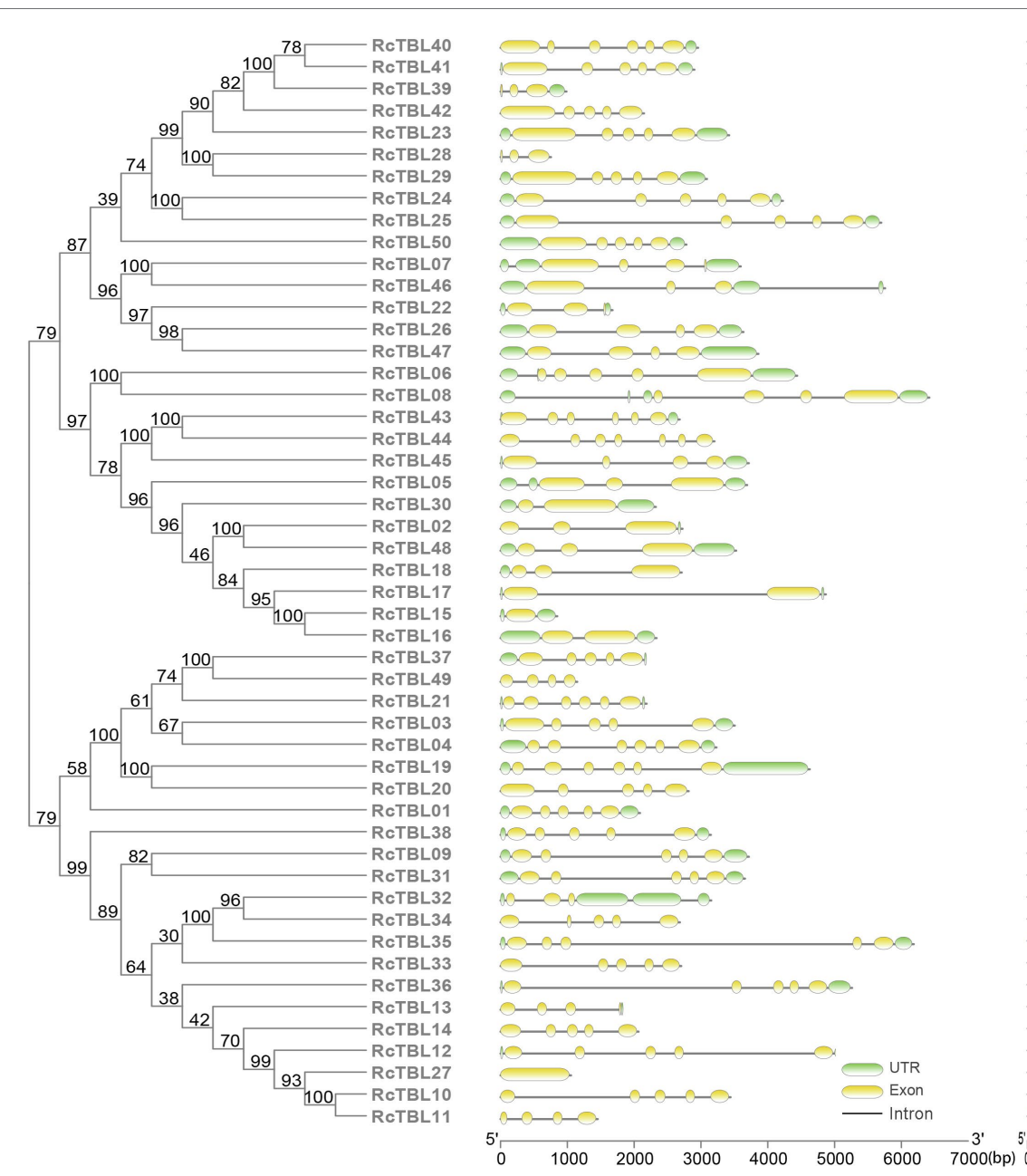

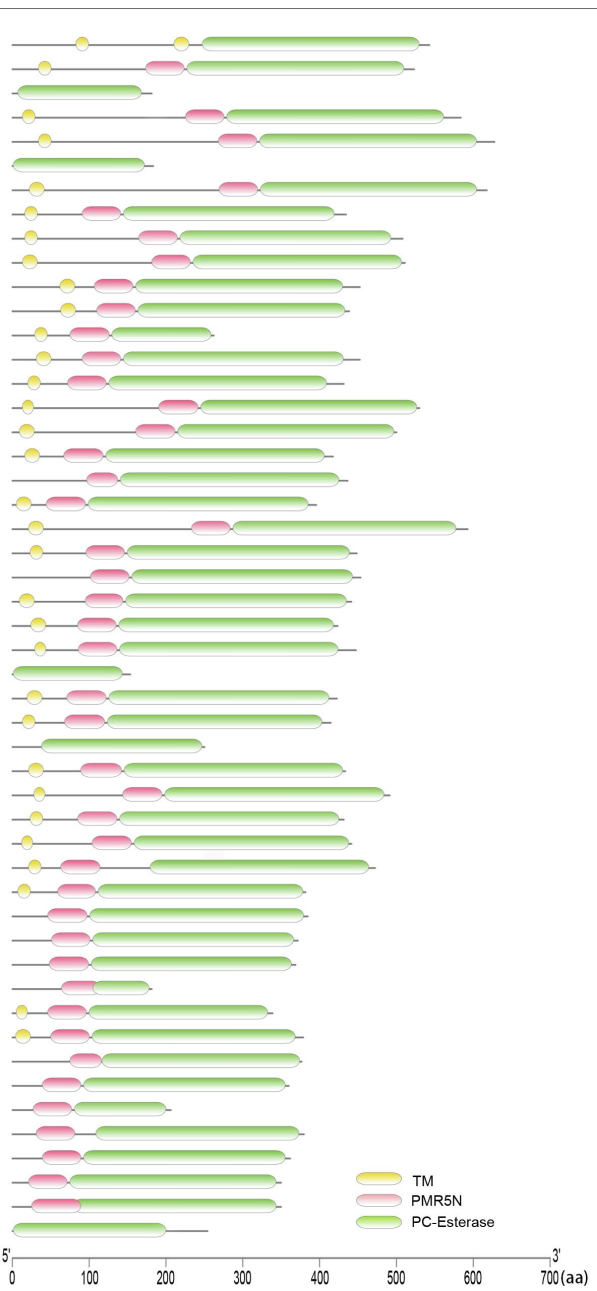

FIGURE 4 | Phylogenetic analyses and gene structures of rose TBL proteins. Complete alignments of all rose TBL proteins were used to construct a phylogenetic tree using the maximum likelihood method. The bootstrap values are indicated on the nodes of the branches. Exon-intron structure of RcTBLs is shown in the middle part of the figure, and the green boxes, yellow boxes, and gray lines represent UTRs, exons, and introns, respectively. Transmembrane and conserved domain of RcTBLs are displayed in the right part of the figure, and the green boxes, yellow boxes, and pink boxes represent PC-Esterase domain, transmembrane domain, and PMR5N domain, respectively. The scale on the bottom is provided as a reference.

The composition of the cell wall is closely correlated with fungal disease resistance, and altered levels of cell wall polysaccharide $O$-acetylation modification can lead to altered plant resistance to fungi. In some cases, a reduction in the level of acetylation enhances plant resistance to pathogens. For instance, Arabidopsis rwa2 showed a $20 \%$ reduction in the degree of cell wall polysaccharide acetylation modification and was more resistant to $B$. cinerea than wild type. In this study, we also found RcTBL16 negatively regulating resistance to gray mold in rose. PMR5, a member of the TBL family, has the same performance in resisting powdery mildew in Arabidopsis (Vogel et al., 2004a), by the O-acetylation modification of homogalacturonan (Chiniquy et al., 2019a). Therefore, we consider that partial rose TBL genes may be involved in the susceptibility of rose to $B$. cinerea through acetylating cell wall.

It is clear that changes in the level of $O$-acetylation modification of cell walls can affect plant resistance or susceptibility to pathogens, but the exact mechanism remains a mystery at present. One hypothesis suggests that alterations in the polysaccharide composition of the cell wall will modify the cell wall integrity (CWI) system, thereby triggering plant defenses and activating specific defense responses (Bacete et al., 2017). Alteration of cell wall xylan acetylation caused by ESK1 impairment was accompanied by an enhanced accumulation of abscisic acid, the constitutive expression of genes encoding antibiotic peptides and enzymes involved in the biosynthesis of tryptophan-derived metabolites, and the accumulation of disease resistance-related secondary metabolites and different osmolites, implying that the damage to CWI triggers defense response of plant (Lugan et al., 2010; Xin et al., 2010; Xu et al., 2014; Escudero et al., 2017). However, it needs to be substantiated by stronger evidence. Overall, more research remains to be done on the certain mechanisms of TBL participant plant-pathogen interactions, but there is no doubt that TBLs in rose is most possibly 
TABLE 4 | Expression patterns of RcTBL genes under infection of $B$. cinerea ${ }^{1}$.

\begin{tabular}{|c|c|c|c|}
\hline Gene $^{2}$ & $\begin{array}{l}\text { Accession } \\
\text { number }\end{array}$ & $\log ^{2}$ ratio $30 \mathrm{hpi}$ & $\log ^{2}$ ratio $48 \mathrm{hpi}^{3}$ \\
\hline RcTBL02 & $\begin{array}{l}\text { RchiOBHm_ } \\
\text { Chr1g0359011 }\end{array}$ & -1.450 & - \\
\hline RcTBL04 & $\begin{array}{l}\text { RchiOBHm_ } \\
\text { Chr1g0371061 }\end{array}$ & -1.14643 & 0.405416 \\
\hline RcTBL05 & $\begin{array}{l}\text { RchiOBHm_ } \\
\text { Chr1g0375151 }\end{array}$ & -1.210 & -0.707 \\
\hline RcTBL06 & $\begin{array}{l}\text { RchiOBHm_ } \\
\text { Chr2g0095401 }\end{array}$ & -0.366 & 1.237 \\
\hline RcTBL09 & $\begin{array}{l}\text { RchiOBHm_ } \\
\text { Chr2g0129071 }\end{array}$ & 0.726 & 1.139 \\
\hline RcTBL12 & $\begin{array}{l}\text { RchiOBHm_ } \\
\text { Chr2g0131441 }\end{array}$ & -1.646 & -1.875 \\
\hline RcTBL16 & $\begin{array}{l}\text { RchiOBHm_ } \\
\text { Chr2g0163771 }\end{array}$ & -2.239 & - \\
\hline RcTBL18 & $\begin{array}{l}\text { RchiOBHm_ } \\
\text { Chr2g0170621 }\end{array}$ & -1.639 & 1.416 \\
\hline RcTBL23 & $\begin{array}{l}\text { RchiOBHm_ } \\
\text { Chr3g0479331 }\end{array}$ & -0.682 & -1.012 \\
\hline RcTBL35 & $\begin{array}{l}\text { RchiOBHm_ } \\
\text { Chr5g0029601 }\end{array}$ & -2.017 & -1.643 \\
\hline RcTBL36 & $\begin{array}{l}\text { RchiOBHm_ } \\
\text { Chr5g0029611 }\end{array}$ & -1.079 & - \\
\hline RcTBL38 & $\begin{array}{l}\text { RchiOBHm_ } \\
\text { Chr5g0070191 }\end{array}$ & -0.505 & -2.356 \\
\hline RcTBL48 & $\begin{array}{l}\text { RchiOBHm_ } \\
\text { Chr7g0195391 }\end{array}$ & -0.361 & -1.129 \\
\hline
\end{tabular}

${ }^{1}$ The log2 transformed expression profiles were obtained from the RNA-seq dataset (Liu et al., 2018).

${ }^{2}$ The RcTBLs undergo duplicate events are marked in bold.

${ }^{3} \mathrm{~A}$ dash '-' means that data are not available.

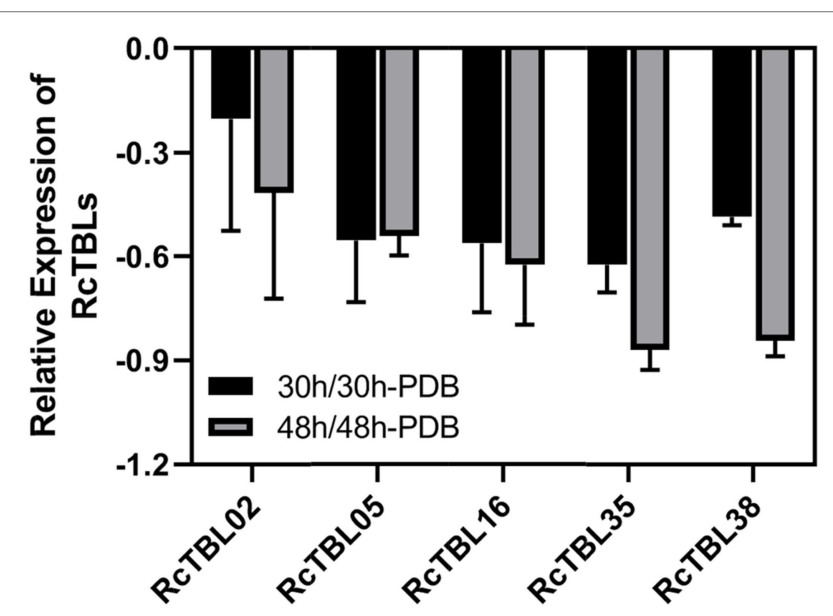

FIGURE 5 | Validation of RNA-Seq results using RT-qPCR. RcUB/2 was used as an internal control. Expression profile data of five RCTBL genes at $30 \mathrm{hpi}$ and $48 \mathrm{hpi}$ after $B$. cinerea inoculation were obtained using RT-qPCR. Values are the means of three technical replicates \pm SD. The used primers are listed in Supplementary Table S1.

as a susceptibility factor for the resistance to gray mold, and this result will also provide clues for rose breeding application (i.e., enhancing persistent plant resistance by silencing or knocking out susceptibility genes).

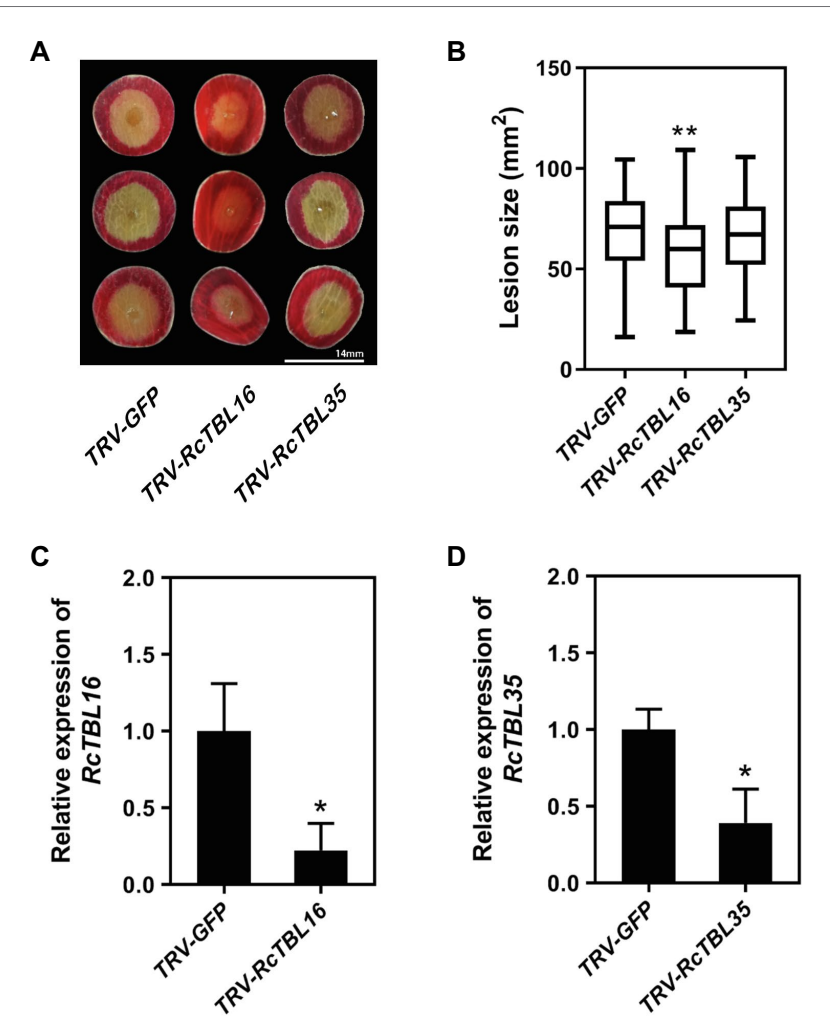

FIGURE 6 | Functional analysis of RcTBL16. (A) Altered B. cinerea resistance upon silencing of RCTBL16 and unaltered $B$. cinerea resistance upon silenced RcTBL35 at $60 \mathrm{hpi}$ post-inoculation. (B) Quantification of $B$. cinerea disease lesions on TRV-RcTBL16, TRV-RcTBL35, and TRV-GFPinoculated rose petal discs. The graph shows the lesion size from one replicate out of four $(n \geq 48)$ with the standard deviation. (C) Quantification of RCTBL16 expression in TRV-RCTBL16 and TRV-GFP-inoculated petal discs. (D) Quantification of RCTBL35 expression in TRV-RcTBL35 and TRV-GFPinoculated petal discs. Statistical analyses were performed using Student's $t$ test; ${ }^{\star} p<0.01,{ }^{*} p<0.05$.

\section{CONCLUSION}

The study performed a genome-wide analysis of RcTBLs, including phylogenetic relationships, collinearity, and expression analysis. A total of 50 non-redundant $R c T B L$ members were identified. The expression analysis showed that transcription regulation of several $R c T B L$ was reduced by $B$. cinerea infection in rose petals. Based on these analyses and VIGS, we further demonstrated that RcTBL16 was engaged in susceptibility of rose to gray mold. The information provided by these results can promote the further functional analysis of RcTBL genes and application in rose disease resistance breeding.

\section{DATA AVAILABILITY STATEMENT}

Publicly available datasets were analyzed in this study. These data can be found at: https://lipm-browsers.toulouse.inra.fr/pub/ RchiOBHm-V2/. 


\section{AUTHOR CONTRIBUTIONS}

$\mathrm{ZZ}$ and YT conceived and designed the research and wrote the paper. YT performed the experiments. YT, SZ, XL, and $\mathrm{ZZ}$ analyzed the data. All the authors have read and approved the final version of the manuscript. All authors contributed to the article and approved the submitted version.

\section{FUNDING}

This study was supported by the National Natural Science Foundation of China (grant number 31772344) to ZZ.

\section{REFERENCES}

Bacete, L., Mélida, H., Miedes, E., and Molina, A. (2017). Plant cell wallmediated immunity: cell wall changes trigger disease resistance responses. Plant J. 93, 614-636. doi: 10.1111/tpj.13807

Bendahmane, M., Dubois, A., Raymond, O., and Bris, M. L. (2013). Genetics and genomics of flower initiation and development in roses. J. Exp. Bot. 64, 847-857. doi: $10.1093 /$ jxb/ers387

Bischoff, V., Nita, S., Neumetzler, L., Schindelasch, D., Urbain, A., Eshed, R., et al. (2010a). TRICHOME BIREFRINGENCE and its homolog AT5G01360 encode plant-specific DUF231 proteins required for cellulose biosynthesis in Arabidopsis. Plant Physiol. 153, 590-602. doi: 10.1104/ pp. 110.153320

Bischoff, V., Selbig, J., and Scheible, W. R. (2010b). Involvement of TBL/DUF231 proteins into cell wall biology. Plant Signaling 5, 1057-1059.

Cao, X., Yan, H., Liu, X., Li, D., Sui, M., Wu, J., et al. (2019). A detached petal disc assay and virus-induced gene silencing facilitate the study of Botrytis cinerea resistance in rose flowers. Hortic. Res. 6:136. doi: 10.1038/ s41438-019-0219-2

Chen, C., Xia, R., Chen, H., and He, Y. (2018). TBtools, a toolkit for biologists integrating various HTS-data handling tools with a user-friendly interface. Mol. Plant 13, 1194-1202. doi: 10.1016/j.molp.2020.06.009

Chiniquy, D., Underwood, W., Corwin, J., Ryan, A., Szemenyei, H., Lim, C. C., et al. (2019a). PMR5, an acetylation protein at the intersection of pectin biosynthesis and defense against fungal pathogens. Plant J. 100, 1022-1035. doi: $10.1111 /$ tpj.14497

Chiniquy, D., Underwood, W., Corwin, J., Ryan, A., Szemenyei, H., Lim, C. C., et al. (2019b). PMR5, an acetylation protein at the intersection of pectin biosynthesis and defense against fungal pathogens. Plant J. 100, 1022-1035. doi: $10.1111 /$ tpj.14497

Escudero, V., Jordá, L., SopeA-Torres, S., Mélida, H., Miedes, E., MuOz-Barrios, A., et al. (2017). Alteration of cell wall xylan acetylation triggers defense responses that counterbalance the immune deficiencies of plants impaired in the $\beta$-subunit of the heterotrimeric G-protein. Plant J. 92, 386-399. doi: 10.1111/ tpj. 13660

Gao, Y., He, C., Zhang, D., Liu, X., and Xu, Z. (2017). Two trichome birefringencelike proteins mediate xylan acetylation, which is essential for leaf blight resistance in rice. Plant Physiol. 173, 470-481. doi: 10.1104/pp.16.01618

Gille, S., de Souza, A., Xiong, G., Benz, M., Cheng, K., Schultink, A., et al. (2011a). O-acetylation of Arabidopsis hemicellulose xyloglucan requires AXY4 or AXY4L, proteins with a TBL and DUF231 domain. Plant Cell 23, 40414053. doi: $10.1105 /$ tpc. 111.091728

Gille, S., De Souza, A., Xiong, G., Benz, M., Cheng, K., Schultink, A., et al. (2011b). O-acetylation of Arabidopsis hemicellulose xyloglucan requires AXY4 or AXY4L, proteins with a TBL and DUF231 domain. Plant Cell 23, 4041-4053. doi: 10.1105/tpc.111.091728

Gleason, M., and Helland, S. (2003). "DISEASE|Botrytis," in Encyclopedia of Rose Science. eds. A. Roberts, T. Debener, and S. Gudin (Amsterdam: Elsevier Academic Press), 144-148.

Keegstra, K. (2010). Plant cell walls. Plant Physiol. 154, 483-486. doi: 10.1104/ pp. 110.161240
It is further supported by the Science and Technology Program of Beijing Municipality (grant number $\mathrm{Z} 181100002518002)$. The funders played no role in study design, data collection and analysis, decision to publish, or preparation of the manuscript.

\section{SUPPLEMENTARY MATERIAL}

The Supplementary Material for this article can be found online at: https://www.frontiersin.org/articles/10.3389/fpls.2021.738880/ full\#supplementary-material

Liu, X., Cao, X., Shi, S., Zhao, N., Li, D., Fang, P., et al. (2018). Comparative RNA-Seq analysis reveals a critical role for brassinosteroids in rose (Rosa hybrida) petal defense against Botrytis cinerea infection. BMC Genet. 19, 1-10. doi: 10.1186/s12863-018-0668-X

Liu, Y., Schiff, M., and Dinesh-Kumar, S. P. (2002). Virus-induced gene silencing in tomato. Plant J. 31, 777-786. doi: 10.1046/j.1365-313X.2002.01394.x

Lugan, R., Niogret, M.-F., Kervazo, L., Larher, F. R., and Bouchereau, A. (2010). Metabolome and water status phenotyping of Arabidopsis under abiotic stress cues reveals new insight into ESK1 function. Plant Cell Environ. Ecol. 32, 95-108. doi: 10.1111/j.1365-3040.2008.01898.x

Manabe, Y., Nafisi, M., Verhertbruggen, Y., Orfila, C., Gille, S., Rautengarten, C., et al. (2011). Loss-of-function mutation of REDUCED WALL ACETYLATION2 in Arabidopsis leads to reduced cell wall ACETYLATION and increased resistance to Botrytis cinerea. Plant Physiol. 155, 1068-1078. doi: $10.1104 /$ pp.110.168989

Manabe, Y., Verhertbruggen, Y., Gille, S., Harholt, J., Chong, S.-L., Pawar, P. M.-A., et al. (2013). Reduced wall acetylation proteins play vital and distinct roles in cell wall o-acetylation in Arabidopsis. Plant Physiol. 163, 1107-1117. doi: 10.1104/pp.113.225193

Schultink, A., Naylor, D., Dama, M., and Pauly, M. (2015). The role of the plant-specific ALTERED XYLOGLUCAN9 protein in Arabidopsis cell wall polysaccharide O-acetylation. Plant Physiol. 167, 1271-U1243. doi: 10.1104/ pp.114.256479

Stranne, M., Ren, Y., Fimognari, L., Birdseye, D., and Sakuragi, Y. (2018a). TBL10 is required for O-acetylation of pectic rhamnogalacturonan-I in Arabidopsis thaliana. Plant J. 96, 772-785. doi: 10.1111/tpj.14067

Stranne, M., Ren, Y., Fimognari, L., Birdseye, D., Yan, J., Bardor, M., et al. (2018b). TBL10 is required for O-acetylation of pectic rhamnogalacturonan-I in Arabidopsis thaliana. Plant J. 96, 772-785. doi: 10.1111/tpj.14067

Urbanowicz, B. R., Pea, M. J., Moniz, H. A., Moremen, K. W., and York, W. S. (2015). Two Arabidopsis proteins synthesize acetylated xylan invitro. Plant J. 80, 197-206. doi: 10.1111/tpj.12643

Vogel, J. P., Raab, T. K., Somerville, C. R., and Somerville, S. C. (2004a). Mutations in PMR5 result in powdery mildew resistance and altered cell wall composition. Plant J. 40:968. doi: 10.1111/j.1365-313X.2004.02264.x

Vogel, J. P., Raab, T. K., Somerville, C. R., and Somerville, S. C. (2004b). Mutations in PMR5 result in powdery mildew resistance and altered cell wall composition. Plant J. 40, 968-978. doi: 10.1111/j.1365-313X. 2004.02264.x

Wang, Y., Tang, H., DeBarry, J. D., Tan, X., Li, J., Wang, X., et al. (2012). MCScanX: a toolkit for detection and evolutionary analysis of gene synteny and collinearity. Nucleic Acids Res. 40:e49. doi: 10.1093/nar/gkr1293

Wu, L., Ma, N., Jia, Y., Zhang, Y., Feng, M., Jiang, C. Z., et al. (2016). An ethylene-induced regulatory module delays flower senescence by regulating cytokinin content. Plant Physiol. 173, 853-862. doi: 10.1104/pp.16.01064

Xin, Z., Mandaokar, A., Chen, J., Last, R. L., and Browse, J. (2010). Arabidopsis ESK1 encodes a novel regulator of freezing tolerance. Plant J. 49, 786-799. doi: 10.1111/j.1365-313X.2006.02994.x

Xiong, G., Cheng, K., and Pauly, M. (2013). Xylan O-acetylation impacts xylem development and enzymatic recalcitrance as indicated by the Arabidopsis mutant tbl29. Mol. Plant 6, 1373-1375. doi: 10.1093/mp/sst014 
Xu, F., Liu, Z., Xie, H., Zhu, J., and Zhang, J. (2014). Increased drought tolerance through the suppression of ESKMO1 gene and Overexpression of CBF-related genes in Arabidopsis. PLoS One 9:e106509. doi: 10.1371/journal.pone.0115300

Yuan, Y., Teng, Q., Zhong, R., Haghighat, M., Richardson, E. A., and Ye, Z.-H. (2016a). Mutations of Arabidopsis TBL32 and TBL33 affect xylan acetylation and secondary wall deposition. PLoS One 11:e0146460. doi: 10.1371/journal. pone. 0146460

Yuan, Y., Teng, Q., Zhong, R., and Ye, Z.-H. (2016b). Roles of Arabidopsis TBL34 and TBL35 in xylan acetylation and plant growth. Plant Sci. 243, 120-130. doi: 10.1016/j.plantsci.2015.12.007

Yuan, Y., Teng, Q., Zhong, R., and Ye, Z.-H. (2016c). TBL3 and TBL31, two Arabidopsis DUF231 domain proteins, are required for 3-O-monoacetylation of xylan. Plant Cell Physiol. 57, 35-45. doi: 10.1093/pcp/pcv172

Zhong, R., Cui, D., Dasher, R. L., and Ye, Z. H. (2018a). Biochemical characterization of rice xylan O-acetyltransferases. Planta 247, 1-10. doi: 10.1007/s00425-018-2882-1

Zhong, R., Cui, D., Phillips, D. R., Richardson, E. A., and Ye, Z.-H. (2020). A group of O-acetyltransferases catalyze xyloglucan backbone acetylation and can alter xyloglucan xylosylation pattern and plant growth when expressed in Arabidopsis. Plant Cell Physiol. 61, 1064-1079. doi: 10.1093/pcp/pcaa031

Zhong, R. Q., Cui, D. T., and Ye, Z. H. (2017a). Regiospecific acetylation of xylan is mediated by a group of DUF231-containing O-acetyltransferases. Plant Cell Physiol. 58, 2126-2138. doi: 10.1093/pcp/pcx147

Zhong, R., Cui, D., and Ye, Z.-H. (2018b). Members of the DUF231 family are O-acetyltransferases catalyzing 2-O- and 3-O-acetylation of mannan. Plant Cell Physiol. 59, 2339-2349. doi: 10.1093/pcp/pcy159

Zhong, R., Cui, D., and Ye, Z.-H. (2018c). Xyloglucan O -acetyltransferases from Arabidopsis thaliana and Populus trichocarpa catalyze acetylation of fucosylated galactose residues on xyloglucan side chains. Planta 248, 11591171. doi: $10.1007 / \mathrm{s} 00425-018-2972-0$
Zhong, R. Q., Cui, D. T., and Ye, Z. H. (2018e). Xyloglucan O-acetyltransferases from Arabidopsis thaliana and Populus trichocarpa catalyze acetylation of fucosylated galactose residues on xyloglucan side chains. Planta 248, 11591171. doi: 10.1007/s00425-018-2972-0

Zhong, R., Cui, D., Ye, Z.-H., and Zabotina, O. A. (2018d). A group of Populus trichocarpa DUF231 proteins exhibit differential O-acetyltransferase activities toward xylan. PLoS One 13:e0194532. doi: 10.1371/journal.pone.0194532

Zhong, R., Dongtao, C., and Zheng-Hua, Y. (2017b). Regiospecific acetylation of xylan is mediated by a group of DUF231-containing O-acetyltransferases. Plant Cell Physiol. 58, 2126-2138. doi: 10.1093/pcp/pcx147

Zlesak, D. C. (2007). "Rose," in Flower Breeding and Genetics. ed. N. O. Anderson (Dordrecht: Springer), 695-740.

Conflict of Interest: The authors declare that the research was conducted in the absence of any commercial or financial relationships that could be construed as a potential conflict of interest.

Publisher's Note: All claims expressed in this article are solely those of the authors and do not necessarily represent those of their affiliated organizations, or those of the publisher, the editors and the reviewers. Any product that may be evaluated in this article, or claim that may be made by its manufacturer, is not guaranteed or endorsed by the publisher.

Copyright (c) 2021 Tian, Zhang, Liu and Zhang. This is an open-access article distributed under the terms of the Creative Commons Attribution License (CC BY). The use, distribution or reproduction in other forums is permitted, provided the original author(s) and the copyright owner(s) are credited and that the original publication in this journal is cited, in accordance with accepted academic practice. No use, distribution or reproduction is permitted which does not comply with these terms. 